\title{
Role of the Immune Tolerance-Inducing Molecule Indoleamine 2,3-Dioxygenase in Gynecologic Cancers
}

Kazuhiko Ino*, Yuko Tanizaki, Aya Kobayashi, Saori Toujima, Yasushi Mabuchi and Sawako Minami

Department of Obstetrics and Gynecology, Wakayama Medical University, Wakayama, Japan

\begin{abstract}
Immune escape and acquisition of tolerance by tumor cells are essential to cancer growth and progression. Therefore, considerable attention has been paid to overcoming the immune resistance of tumors as a novel strategy for cancer therapy. This review focuses on the tryptophan-catabolizing and immunoregulatory enzyme indoleamine 2,3-dioxygenase (IDO), and its functional role in gynecologic cancers, such as endometrial cancer, ovarian cancer, cervical cancer, and vulvar cancer. IDO induces tolerance to the host immune surveillance through suppressing the proliferation of effector T-cells or natural killer cells and their killer functions within the tumor microenvironment. In gynecologic cancers, IDO is highly expressed in more than half of cases, and tumoral IDO expression is correlated with advanced surgical stage and impaired patient survival. In preclinical studies in mice, an IDO inhibitor 1-methyltryptophan suppresses tumor growth and peritoneal dissemination, and increases the efficacy of chemotherapeutic agents. In summary, IDO is a novel prognostic indicator for endometrial, ovarian, cervical, and vulvar cancers. IDO inhibition may be a promising strategy to restore host anti-tumor immunity and to enhance the anti-tumor potential of current chemotherapy, radiotherapy, and immunotherapy for gynecologic cancers.
\end{abstract}

Keywords: Endometrial cancer; Ovarian cancer; Cervical cancer; Vulvar cancer; Immune tolerance; Indoleamine 2,3-dioxygenase; Immunotherapy; Survival

Abbreviations: IDO: Indoleamine 2,3-dioxygenase; 1-MT: 1-methyltryptophan; TIL: Tumor-Infiltrating Lymphocyte; Treg: Regulatory T cell; NK cell: Natural Killer cell

\section{Introduction}

Gynecologic cancer mainly consists of three major tumors; endometrial carcinoma, ovarian carcinoma, and uterine cervical carcinoma, and also includes vulvar carcinoma. Most patients with International Federation of Gynecology and Obstetrics (FIGO) stage I-II early-stage gynecologic cancer achieve a favorable clinical outcome with surgery alone or with surgery plus postoperative adjuvant chemotherapy and/or radiotherapy. However, patients with FIGO stage III-IV advanced disease or recurrence remain to show the poor long-term survival. Therefore, in addition to conventional surgery, chemotherapy and radiotherapy, novel therapeutic strategies, such as immunotherapy and molecular-targeted therapy, are needed to further improve the survival of patients with advanced disease.

Immunotherapy has demonstrated promising results in basic and preclinical animal studies [1], and there have been several clinical trials in gynecologic cancer using immunologic modalities [2,3]. However, clinical applications have shown only limited efficacy [4], and this may be mainly attributed to tumor-induced immunosuppression. Therefore, much attention has been paid for understanding and overcoming the immune resistance mechanisms [5-9]. Recent studies have shown that indoleamine 2,3-dioxygenase (IDO) is one of the molecules involved in this tumor-induced immunosuppression [10-12]. In this review, we focus on the immunoregulatory enzyme IDO, and overview the recent studies

\section{Role of IDO in Tumor-induced Immune Tolerance}

Tumors are known to successfully escape the host immune surveillance, and this acquisition of immune tolerance is essential to cancer growth and progression. In various human cancers, multiple tumor-inducing immunosuppressive mechanisms have been demonstrated [5-9,13-15]; the down-regulation of Human Leukocyte Antigen (HLA) class I, loss of tumor antigens, lack of costimulatory signals, production of immunosuppressive cytokines and prostaglandin E2, induction of immunosuppressive host immune cells including regulatory T cells (Treg), Myeloid-Derived Suppressor Cells (MDSC), and tumor-associated macrophage, and expression of immunosuppressive molecules such as Fas ligand and programmed cell death 1 ligand 1 (PD-L1). In addition, recent studies have suggested that IDO is involved in tumoral immune tolerance [12].

IDO is an intracellular enzyme that catalyzes tryptophan at the initial and rate-limiting step [16]. Evidence for an immunosuppressive function of IDO was first documented in the mouse placenta, where IDO prevents rejection of the allogeneic fetus during pregnancy [17]. Subsequent studies have clarified the mechanisms of IDO immunosuppression in tumors. First, IDO expressed by tumor cells depletes tryptophan locally and produces a toxic tryptophan catabolite kynurenine, which causes growth arrest and the apoptosis of effector T-cells or natural killer (NK) cells that are extremely sensitive to tryptophan shortage, and also suppresses their killer functions $[12,18$ 20]. Secondly, IDO expressed by antigen-presenting Dendritic Cells (DCs) within tumor-draining lymph nodes induces tolerance to tumor-derived antigens [21]. Lastly, IDO expressed by plasmacytoid DCs plays a critical role in conversion of $\mathrm{CD}^{+} \mathrm{CD} 25^{-} \mathrm{T}$ cells into

${ }^{*}$ Corresponding author: Kazuhiko Ino, MD, PhD, Professor and Chairman, Department of Obstetrics and Gynecology, Wakayama Medical University, 811-1 Kimiidera, Wakayama 641-0012, Japan, Tel: 81-(0)73-441-0631; Fax: 81-(0)73445-1161; E-mail: kazuino@wakayama-med.ac.jp

Received March 29, 2012; Accepted April 19 2012; Published April 21, 2012

Citation: Ino K, Tanizaki Y, Kobayashi A, Toujima S, Mabuchi Y, et al. (2012) Role of the Immune Tolerance-Inducing Molecule Indoleamine 2,3-Dioxygenase in Gynecologic Cancers. J Cancer Sci Ther S13:004. doi:10.4172/1948-5956.S13001

Copyright: (c) 2012 Ino K, et al. This is an open-access article distributed under the terms of the Creative Commons Attribution License, which permits unrestricted use, distribution, and reproduction in any medium, provided the original author and source are credited. 
Citation: Ino K, Tanizaki Y, Kobayashi A, Toujima S, Mabuchi Y, et al. (2012) Role of the Immune Tolerance-Inducing Molecule Indoleamine 2,3-Dioxygenase in Gynecologic Cancers. J Cancer Sci Ther S13:004. doi:10.4172/1948-5956.S13-001

Page 2 of 5

$\mathrm{CD}^{+} \mathrm{CD} 25^{+}$Foxp $^{+}$regulatory $\mathrm{T}$ (Treg) cells, directly activating mature Tregs $[22,23]$. These findings suggest that IDO, in cooperation with Treg (and possibly MDSC and immunosuppressive cytokines), induces the immune tolerogenic microenvironment, which leads to tumor progression (Figure 1).

\section{IDO Expression and Function in Gynecologic Cancers}

In human cancer, Uyttenhove et al. [12] first demonstrated that IDO was expressed in various human cancer tissues. Subsequent studies have shown that IDO expression is correlated with disease progression or poor clinical outcomes in various histologic cancer types [24]. In gynecologic cancers including endometrial, ovarian, cervical, and vulvar carcinomas, associations of IDO expression with tumor progression or clinical outcomes have been extensively studied by the authors and others. The results of their studies are summarized in Table 1.

\section{Endometrial cancer}

In endometrial cancer, immunohistochemical analysis by Ino et al. [25] demonstrated that high IDO expression in tumor cells was found in 37 (46.3\%) of the 80 cases, and was positively correlated with surgical stage, myometrial invasion, lymph-vascular space involvement and lymph node metastasis. Patients with high IDO expression had significantly impaired overall survival (OS) and Progression-Free Survival (PFS) compared to patients with no or weak expression of IDO. In their report, IDO expression was an independent prognostic factor for impaired PFS on multivariate analysis. These results indicate that IDO is a novel and reliable prognostic indicator for endometrial cancer.

Recent reports have shown the impact of tumor-infiltrating lymphocytes (TIL) on disease progression and clinical outcome, and suggested that TIL could be a surrogate marker for the immunological status within the tumor microenvironments [26,27]. In endometrial cancer, Ino et al. [28] showed that IDO expression is correlated with reduced numbers of $\mathrm{CD} 8^{+}$TILs and $\mathrm{CD} 57^{+} \mathrm{NK}$ cells, and high IDO expression with reduced TIL count is an independent prognostic factor for impaired survival. These findings suggest that IDO expression is associated with poor clinical outcome via suppression of TIL and/or NK cells within the tumor microenvironment.

Functional role of IDO in human endometrial cancer was first studied by Yoshida et al. [29] using a xenograft mouse model. In their report, a rapid tumor growth and decreased NK cell count and lysis activity were observed in IDO-overexpreesing endometrial cancertransplanted mice. Furthermore, administration of the IDO inhibitor 1-methyltryptophan (1-MT) in combination with paclitaxel in mice potentiated the anti-tumor effect of paclitaxel, resulting in significantly prolonged survival. These data suggest that IDO induces tumor progression through inhibiting host NK activity, and targeting IDO may be a novel therapeutic strategy for endometrial cancer.

\section{Ovarian cancer}

In ovarian cancer, Okamoto et al. [30] first reported that IDO was over expressed in paclitaxel-resistant ovarian cancer tissues in a gene expression profiling study using microarrays, and that patients with diffuse IDO expression have poor clinical outcomes in stage III-IV serous-type ovarian cancer. Similarly, another report demonstrated a relationship between IDO expression and impaired OS for advanced serous ovarian carcinoma, but not for other histological types [31] Recent immunohistochemical study by Inaba et al. [32] using 60 ovarian cancer samples demonstrated that high IDO expression was found in over $70 \%$ cases with stage II-IV advanced diseases, and was significantly correlated with a low number of $\mathrm{CD} 8^{+} \mathrm{TIL}$ and impaired OS/PFS. These findings suggest that IDO acts as a prognostic indicator for ovarian cancer.

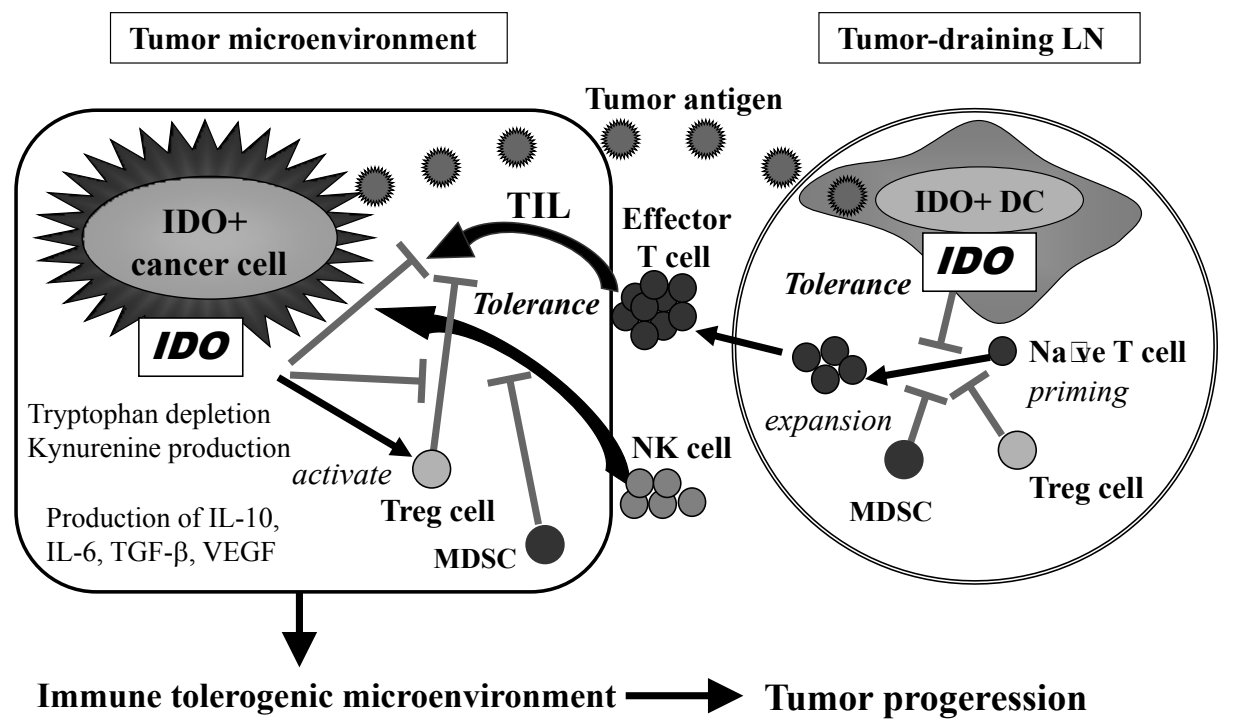

Figure 1: Possible mechanisms of IDO-induced immune tolerance within the tumor microenvironment. IDO expressed by antigen-presenting dendritic cells within tumor-draining lymph nodes induces tolerance to tumor-derived antigens, while IDO expressed by tumor cells within the tumor microenvironment blocks effectors of adaptive immunity (CD8+ T-cells) and innate immunity (NK cells), in cooperation with Treg, MDSC, and immunosuppressive cytokines. This immune tolerogenic microenvironment leads to tumor growth and progression.

IDO: Indoleamine 2,3-dioxygenase; LN: Lymph node; DC: Dendritic cell; NK: Natural killer cell; TIL: Tumor-infiltrating lymphocyte; Treg: Regulatory T cell; MDSC: Myeloid-derived suppressor cell; IL-10: Interleukine-10; IL-6: Interleukine-6; TGF- $\beta$ : Transforming growth factor- $\beta$; VEGF: Vascular endothelial growth factor. 
Citation: Ino K, Tanizaki Y, Kobayashi A, Toujima S, Mabuchi Y, et al. (2012) Role of the Immune Tolerance-Inducing Molecule Indoleamine 2,3-Dioxygenase in Gynecologic Cancers. J Cancer Sci Ther S13:004. doi:10.4172/1948-5956.S13-001

Page 3 of 5

Table 1: Association of IDO expression with tumor progression and clinical outcome in gynecologic cancers.

\begin{tabular}{|c|c|c|}
\hline Type of cancer & Effects of IDO in experimental models & Correlation of IDO expression with clinical outcome \\
\hline [References] & (Methods of evaluation) & (Methods of evaluation, $n$ = sample number) \\
\hline Endometrial cancer & N.D. & Impaird OS and PFS in stage I-IV patients \\
\hline [25] & & $(\mathrm{IHC}, \mathrm{n}=80)$ \\
\hline Endometrial cancer & N.D. & Reduced infitration of CD8 + TIL and CD57 + NK \\
\hline [28] & & $(\mathrm{IHC}, \mathrm{n}=65)$ \\
\hline Endometrial cancer & Induces tumor growth and inhibits NK & N.D. \\
\hline [29] & (in tumor-xenografted mice) & \\
\hline Ovarian cancer & Increases intraperitoneal dissemination & Reduced CD8 + TIL and impaired OS and PFS \\
\hline [32] & (in tumor-xenografted mice) & $(\mathrm{IHC}, \mathrm{n}=60)$ \\
\hline Ovarian cancer & Correlates with chemoresistance to paclitaxel & Impaired OS in stage III-IV serous adenocarcinoma \\
\hline$[30]$ & (microarray gene profiling and PCR) & $(\mathrm{IHC}, \mathrm{n}=24)$ \\
\hline Ovarian cancer & N.D. & Impaired OS in stage III-IV serous adenocarcinoma \\
\hline [31] & & $(\mathrm{IHC}, \mathrm{n}=33)$ \\
\hline Ovarian cancer & Suppresses T-cell proliferation & N.D. \\
\hline$[35]$ & (in vitro) & \\
\hline Ovarian cancer & Increases tumor growth and inhibits NK & N.D. \\
\hline [33] & (in tumor-xenografted mice) & \\
\hline Cervical cancer & N.D. & Impaird OS and DFS in stage IB-IIB cervical cancer \\
\hline [37] & & $(\mathrm{IHC}, \mathrm{n}=112)$ \\
\hline Cervical cancer & N.D. & Progression from $\mathrm{CIN} 2 / 3$ to microinvasive carcinoma \\
\hline$[38]$ & & $(\mathrm{IHC}, \mathrm{n}=46)$ \\
\hline Vulvar cancer & N.D. & Impaird OS in vulvar squamous cell carcinoma \\
\hline [39] & & $(\mathrm{IHC}, \mathrm{n}=76)$ \\
\hline Vulvar cancer & N.D. & No association with the number of CD8 + TIL or Treg \\
\hline [40] & & $(\mathrm{IHC}, \mathrm{n}=286)$ \\
\hline
\end{tabular}

N.D: Not Done; OS: Overall Survival; PFS: Progression-free Survival; DFS: Disease-free Survival; IHC: Immunohistochemistry; TIL: Tumor-infiltrating Lymphocyte; NK: Natural Killer Cell; Treg: Regulatory T cell; CIN: Cervical Intraepithelial Neoplasia

To clarify the functional role of IDO in ovarian cancer progression, Inaba et al. [32] examined the behavior of IDO-overexpressing human ovarian cancer cells in vivo using a tumor-xenografted nude mouse model. In their report, increased peritoneal tumor dissemination was shown in IDO-overexpressing tumor intraperitoneally-transplanted mice. This effect was abrogated by administration of the IDO inhibitor 1-MT. These findings are consistent with another study showing a rapid tumor growth with reduced NK cell accumulation in IDOexpressing human ovarian cancer-transplanted nude mice [33]. Similarly, the IDO-induced tumor progressive effects were inhibited by 1-MT or IDO downregulation by short hairpin RNA targeting IDO $[33,34]$. Furthermore, a recent report by Qian et al. [35] has shown that IDO-positive human ovarian cancer cells suppress T-cell proliferation in vitro. Taken together, it is suggested that IDO enhances the ovarian cancer progression through induction of an immune tolerogenic tumor microenvironment against host effector T cell or NK cell attack [36].

\section{Cervical cancer}

IDO expression in cervical cancer and its association with clinicopathological factors and survival were immunohistochemically analyzed by Inaba et al. [37] in 112 stage IB-IIB patients treated with radical hysterectomy. In their study, IDO was diffusely expressed in tumor cells in $29(26 \%)$ cases and focally expressed at the invasive front in $29(26 \%)$ cases, and the IDO expression was positively correlated with clinical stage and lymph node metastasis. Patients with diffuse IDO expression had significantly reduced OS and Disease-Free Survival (DFS). These findings suggest that IDO may be a post-operative prognostic indicator for cervical cancer. In addition, Nakamura et al. [38] reported that IDO was focally expressed in cervical intraepithelial neoplasia (CIN) 2 to 3 and that its expression was increased in microinvasive cancer, but absent in the normal cervical epithelium and CIN 1, suggesting the involvement of IDO in the progression of cervical neoplasia to invasive cervical cancer. 
Citation: Ino K, Tanizaki Y, Kobayashi A, Toujima S, Mabuchi Y, et al. (2012) Role of the Immune Tolerance-Inducing Molecule Indoleamine 2,3-Dioxygenase in Gynecologic Cancers. J Cancer Sci Ther S13:004. doi:10.4172/1948-5956.S13-001

\section{Vulvar cancer}

IDO expression in vulvar Squamous Cell Carcinoma (SCC) has recently been reported [39,40]. Sznurkowski et al. [39] evaluated the immunohistochemical expression of IDO and its impact on the recruitment of Foxp3 $3^{+}$Tregs within cancer nests in 76 patients with vulvar SCC. In their report, high IDO expression was associated with worse overall survival, and IDO expression was an independent prognostic factor for vulvar cancer, while the degree of intratumoral Treg infiltrates was not correlated with IDO expression or survival. In contrast, another immunohistochemical study in 286 vulvar cancer patients showed that IDO expression was present in $50.4 \%$ of the cases, although its expression was not associated with the number of intratumoral CD8 ${ }^{+}$TILs and Foxp3 $3^{+}$Tregs, or patient survival [40].

\section{Clinical Perspective of IDO-targeted Therapy}

To increase the efficacy of chemotherapy, radiotherapy, and immunotherapy, and further improve patient survival, overcoming the tumor-induced immune tolerance is needed. Therefore, IDOtargeted therapy to restore host anti-tumor immunity may have clinical potential $[41,42]$. Many data obtained from preclinical models demonstrate that IDO inhibition by 1-MT can significantly enhance the anti-tumor activity of various chemotherapeutic agents not only in murine tumors [43,44], but also in human gynecologic cancers [29,32]. Most preclinical studies in mice have used 1-methyl-D-tryptophan (D-1-MT), because the D-stereoisomer of 1-MT was shown to be more effective in reversing $\mathrm{T}$-cell suppression and more efficacious as an anticancer agent when compared to the L-isomer (L-1-MT) in tumorbearing mice [44]. Thus, D-1-MT was initially selected for clinical trials and is currently being used in a phase I study in patients with relapsed or refractory solid tumors to determine the safety and efficacy of its administration at doses from 200 to $2000 \mathrm{mg}$ daily [45,46].

In contrast, recent reports have demonstrated the superiority of L-1-MT. L-1-MT, but not D-1-MT, restored the T-cell proliferation arrest induced by IDO-expressing ovarian cancer cells [35]. Consistently, only L-1-MT, but not D-1-MT, was able to block IDO activity in human dendritic cells [47]. Recent studies have shown that a novel IDO isoform IDO2 is also expressed in human tumors and that IDO1 is the preferential target of L-1-MT, while D-1-MT preferentially inhibits IDO2 [47-50]. In addition, one report showed that D-1-MT upregulates IDO1 expression in some human cancer cells and could have off-target effects, which should be carefully considered in future clinical trials with D-1-MT [51]. Besides 1-MT, a newly discovered potent IDO inhibitor hydroxyamidine has recently been reported to have anti-tumor effects with strong IDO1-inhibiting activity in vitro and in preclinical animal models [52,53]. Further studies are necessary to select the optimal IDO inhibitors in future clinical trials for gynecologic cancers.

\section{Conclusion}

IDO induces immune tolerance within the tumor microenvironment. Tumoral IDO expression is found in more than half of gynecologic cancer patients with advanced staged disease, and is closely correlated with impaired patient survival. Furthermore, IDO overexpression enhances tumor progression and IDO inhibition by $1-\mathrm{MT}$ in combination with chemotherapeutic agents results in potentiated anti-tumor efficacy and prolonged survival in preclinical studies. These findings lead to future clinical trials of IDO-targeted therapies for gynecologic cancers to enhance the antitumor efficacy of current chemotherapy, radiotherapy and immunotherapy.

\section{Conflict of Interest Statement}

The authors declare that there are no conflicts of interest.

\section{References}

1. Rosenberg SA (2001) Progress in human tumour immunology and immunotherapy. Nature 411: 380-384.

2. Sabbatini P, Odunsi K (2007) Immunologic approaches to ovarian cancer treatment. J Clin Oncol 25: 2884-2893.

3. Brooks N, Pouniotis DS (2009) Immunomodulation in endometrial cancer. Int $J$ Gynecol Cancer 19: 734-740

4. Rosenberg SA, Yang JC, Restifo NP (2004) Cancer immunotherapy: moving beyond current vaccines. Nat Med 10: 909-915.

5. Zou W (2005) Immunosuppressive networks in the tumour environment and their therapeutic relevance. Nat Rev Cancer 5: 263-274.

6. Whiteside TL (2006) Immune suppression in cancer: effects on immune cells mechanisms and future therapeutic intervention. Semin Cancer Biol 16: 3-15.

7. Gajewski TF (2006) Identifying and overcoming immune resistance mechanisms in the melanoma tumor microenvironment. Clin Cancer Res 12: 2326s-2330s.

8. Zitvogel L, Tesniere A, Kroemer G (2006) Cancer despite immunosurveillance: immunoselection and immunosubversion. Nat Rev Immunol 6: 715-727.

9. Yigit R, Massuger LF, Figdor CG, Torensma R (2010) Ovarian cancer creates a suppressive microenvironment to escape immune elimination. Gynecol Oncol 117: 366-372.

10. Munn DH, Mellor AL (2007) Indoleamine 2,3-dioxygenase and tumor-induced tolerance. J Clin Invest 117: 1147-1154

11. Katz JB, Muller AJ, Prendergast GC (2008) Indoleamine 2,3-dioxygenase in T-cell tolerance and tumoral immune escape. Immunol Rev 222: 206-221.

12. Uyttenhove C, Pilotte L, Théate I, Stroobant V, Colau D, et al. (2003) Evidence for a tumoral immune resistance mechanism based on tryptophan degradation by indoleamine 2,3-dioxygenase. Nat Med 9: 1269-1274.

13. Curiel TJ, Coukos G, Zou L, Alvarez X, Cheng P, et al. (2004) Specific recruitment of regulatory $T$ cells in ovarian carcinoma fosters immune privilege and predicts reduced survival. Nat Med 10: 942-949.

14. Nagaraj S, Gabrilovich DI (2008) Tumor escape mechanism governed by myeloid-derived suppressor cells. Cancer Res 68: 2561-2563.

15. Hamanishi J, Mandai M, Iwasaki M, Okazaki T, Tanaka Y, et al. (2007) Programmed cell death 1 ligand 1 and tumor-infiltrating CD8+ T lymphocytes are prognostic factors of human ovarian cancer. Proc Natl Acad Sci USA 104: 3360-3365.

16. Takikawa $O$ (2005) Biochemical and medical aspects of the indoleamine 2,3-dioxygenase-initiated L-tryptophan metabolism. Biochem Biophys Res Commun 338: 12-19.

17. Munn DH, Zhou M, Attwood JT, Bondarev I, Conway SJ, et al. (1998) Prevention of allogeneic fetal rejection by tryptophan catabolism. Science 281: 1191-1193.

18. Frumento G, Rotondo R, Tonetti M, Damonte G, Benatti U, et al. (2002) Tryptophan-derived catabolites are responsible for inhibition of $T$ and natura killer cell proliferation induced by indoleamine 2,3-dioxygenase. J Exp Med 196: 459-468

19. Terness P, Bauer TM, Röse L, Dufter C, Watzlik A, et al. (2002) Inhibition of allogeneic $T$ cell proliferation by indoleamine 2,3-dioxygenase-expressing dendritic cells: mediation of suppression by tryptophan metabolites. J Exp Med 196: 447-457.

20. Della Chiesa M, Carlomagno S, Frumento G, Balsamo M, Cantoni C, et al (2006) The tryptophan catabolite L-kynurenine inhibits the surface expression of NKp46- and NKG2D-activating receptors and regulates NK-cell function. Blood 108: 4118-4125.

21. Munn DH, Sharma MD, Hou D, Baban B, Lee JR, et al. (2004) Expression of indoleamine 2,3-dioxygenase by plasmacytoid dendritic cells in tumor-draining lymph nodes. J Clin Invest 114: 280-290. 
Citation: Ino K, Tanizaki Y, Kobayashi A, Toujima S, Mabuchi Y, et al. (2012) Role of the Immune Tolerance-Inducing Molecule Indoleamine 2,3-Dioxygenase in Gynecologic Cancers. J Cancer Sci Ther S13:004. doi:10.4172/1948-5956.S13-001

22. Sharma MD, Baban B, Chandler P, Hou DY, Singh N, et al. (2007) Plasmacytoid dendritic cells from mouse tumor-draining lymph nodes directly activate mature Tregs via indoleamine 2,3-dioxygenase. J Clin Invest 117: 2570-2582.

23. Chen W, Liang X, Peterson AJ, Munn DH, Blazar BR (2008) The indoleamine 2,3-dioxygenase pathway is essential for human plasmacytoid dendritic cellinduced adaptive T regulatory cell generation. J Immunol 181: 5396-5404

24. Godin-Ethier J, Hanafi LA, Piccirillo CA, Lapointe R (2011) Indoleamine 2,3-dioxygenase expression in human cancers: clinical and immunologic perspectives. Clin Cancer Res 17: 6985-6991.

25. Ino K, Yoshida N, Kajiyama H, Shibata K, Yamamoto E, et al. (2006) Indoleamine 2,3-dioxygenase is a novel prognostic indicator for endometrial cancer. Br J Cancer 95: 1555-1561.

26. Zhang L, Conejo-Garcia JR, Katsaros D, Gimotty PA, Massobrio M, et al. (2003) Intratumoral T cells, recurrence, and survival in epithelial ovarian cancer. N Engl J Med 348: 203-213.

27. Nelson BH (2008) The impact of T-cell immunity on ovarian cancer outcomes. Immunol Rev 222: 101-116.

28. Ino K, Yamamoto E, Shibata K, Kajiyama H, Yoshida N, et al. (2008) Inverse correlation between tumoral indoleamine 2,3-dioxygenase expression and tumor-infiltrating lymphocytes in endometrial cancer: its association with disease progression and survival. Clin Cancer Res 14: 2310-2317.

29. Yoshida N, Ino K, Ishida $Y$, Kajiyama H, Yamamoto E, et al. (2008) Overexpression of indoleamine 2,3-dioxygenase in human endometrial carcinoma cells induces rapid tumor growth in a mouse xenograft model. Clin Cancer Res 14: 7251-7259.

30. Okamoto A, Nikaido T, Ochiai K, Takakura S, Saito M, et al. (2005) Indoleamine 2,3-dioxygenase serves as a marker of poor prognosis in gene expression profiles of serous ovarian cancer cells. Clin Cancer Res 11: 6030-6039.

31. Takao M, Okamoto A, Nikaido T, Urashima M, Takakura S, et al. (2007) Increased synthesis of indoleamine-2,3-dioxygenase protein is positively associated with impaired survival in patients with serous-type, but not with other types of, ovarian cancer. Oncol Rep 17:1333-1339.

32. Inaba T, Ino K, Kajiyama H, Yamamoto E, Shibata K, et al. (2009) Role of the immunosuppressive enzyme indoleamine 2,3-dioxygenase in the progression of ovarian carcinoma. Gynecol Oncol 115: 185-192.

33. Nonaka H, Saga Y, Fujiwara H, Akimoto H, Yamada A, et al. (2011) Indoleamine 2,3-dioxygenase promotes peritoneal dissemination of ovarian cancer through inhibition of natural killercell function and angiogenesis promotion. Int $\mathrm{J}$ Oncol 38: $113-120$

34. Wang D, Saga Y, Mizukami H, Sato N, Nonaka H, et al. (2012) Indoleamine2,3-dioxygenase, an immunosuppressive enzyme that inhibits natural killer cell function, as a useful target for ovarian cancer therapy. Int J Oncol 40: 929-934.

35. Qian F, Villella J, Wallace PK, Mhawech-Fauceglia P, Tario JD Jr, et al. (2009) Efficacy of levo-1-methyl tryptophan and dextro-1-methyl tryptophan in reversing indoleamine-2,3-dioxygenase-mediated arrest of T-cell proliferation in human epithelial ovarian cancer. Cancer Res 69: 5498-5504.

36. Ino K (2011) Indoleamine 2,3-dioxygenase and immune tolerance in ovarian cancer. Curr Opin Obstet Gynecol 23: 13-18.

37. Inaba T, Ino K, Kajiyama H, Shibata K, Yamamoto E, et al. (2010) Indoleamine 2,3-dioxygenase expression predicts impaired survival of invasive cervical cancer patients treated with radical hysterectomy. Gynecol Oncol 117: 423428.

38. Nakamura T, Shima T, Saeki A, Hidaka T, Nakashima A, et al. (2007) Expression of indoleamine 2, 3-dioxygenase and the recruitment of Foxp3expressing regulatory $\mathrm{T}$ cells in the development and progression of uterine cervical cancer. Cancer Sci 98: 874-881.

39. Sznurkowski JJ, Żawrocki A, Emerich J, Sznurkowska K, Biernat W (2011) Expression of indoleamine 2,3-dioxygenase predicts shorter survival in patients with vulvar squamous cell carcinoma (vSCC) not influencing on the recruitment of FOXP3-expressing regulatory T cells in cancer nests. Gynecol Oncol 122: 307-312.

This article was originally published in a special issue, Tumour Immunity handled by Editor(s). Dr. Richard J. Simpson, University of Melbourne, Australia; Dr. Suresh Koduru, University of Hyderabad, India
40. de Jong RA, Toppen NL, Ten Hoor KA, Boezen HM, Kema IP, et al. (2012) Status of cellular immunity lacks prognostic significance in vulvar squamous carcinoma. Gynecol Oncol 125: 186-193.

41. Löb S, Königsrainer A, Rammensee HG, Opelz G, Terness $P$ (2009) Inhibitors of indoleamine-2,3-dioxygenase for cancer therapy: can we see the wood for the trees? Nat Rev Cancer 9: 445-452.

42. Liu X, Newton RC, Friedman SM, Scherle PA (2009) Indoleamine 2,3-dioxygenase, an emerging target for anti-cancer therapy. Curr Cancer Drug Targets 9: 938-952.

43. Muller AJ, DuHadaway JB, Donover PS, Sutanto-Ward E, Prendergast GC (2005) Inhibition of indoleamine 2,3-dioxygenase, an immunoregulatory target of the cancer suppression gene Bin1, potentiates cancer chemotherapy. Nat Med 11: 312-319.

44. Hou DY, Muller AJ, Sharma MD, DuHadaway J, Banerjee T, et al. (2007) Inhibition of indoleamine 2,3-dioxygenase in dendritic cells by stereoisomers of 1-methyl-tryptophan correlates with antitumor responses. Cancer Res 67 792-801.

45. Soliman HH, Antonia S, Sullivan D, Vanahanian N, Link C (2009) Overcoming tumor antigen anergy in human malignancies using the novel indoleamine 2,3-dioxygenase (IDO) enzyme inhibitor, 1-methyl-D-tryptophan (1MT). J Clin Oncol 27: 15S.

46. http://clinicaltrials.gov/ct2/show/NCT00739609?term=1-methyl-D-tryptophan

47. Lob S, Konigsrainer A, Schafer R, Rammensee HG, Opelz G, et al. (2008) Levo- but not dextro-1-methyl tryptophan abrogates the IDO activity of human dendritic cells. Blood 111: 2152-2154.

48. Löb S, Königsrainer A, Zieker D, Brücher BL, Rammensee HG, et al. (2009) IDO1 and IDO2 are expressed in human tumors: levo- but not dextro-1-methy tryptophan inhibits tryptophan catabolism. Cancer Immunol Immunother 58 153-157.

49. Metz R, Duhadaway JB, Kamasani U, Laury-Kleintop L, Muller AJ, et al. (2007) Novel tryptophan catabolic enzyme IDO2 is the preferred biochemical target of the antitumor indoleamine 2,3-dioxygenase inhibitory compound D-1-methyltryptophan. Cancer Res 67: 7082-7087.

50. Witkiewicz AK, Costantino CL, Metz R, Muller AJ, Prendergast GC, et al. (2009) Genotyping and expression analysis of IDO2 in human pancreatic cancer: a novel, active target. J Am Coll Surg 208: 781-787.

51. Opitz CA, Litzenburger UM, Opitz U, Sahm F, Ochs K, et al. (2011) The indoleamine-2,3-dioxygenase (IDO) inhibitor 1-methyl-D-tryptophan upregulates IDO1 in human cancer cells. PLoS One 6: e19823.

52. Koblish HK, Hansbury MJ, Bowman KJ, Yang G, Neilan CL, et al. (2010) Hydroxyamidine inhibitors of indoleamine-2,3-dioxygenase potently suppress systemic tryptophan catabolism and the growth of IDO-expressing tumors. Mol Cancer Ther 9: 489-498.

53. Liu X, Shin N, Koblish HK, Yang G, Wang Q, et al. (2010) Selective inhibition of IDO1 effectively regulates mediators of antitumor immunity. Blood 115: 3520 3530 